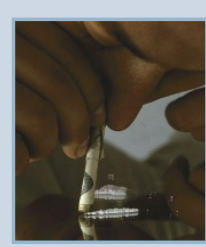

p1007 Tricky Fix: A vaccine for cocaine addiction poses ethical dilemmas.

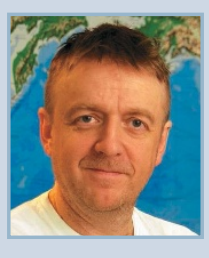

p1009 Bohemian brain: Neuroscientist John Hardy bucks the trends.

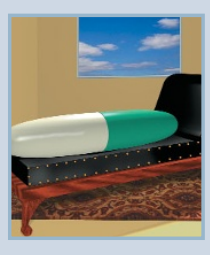

p1010 Better than Prozac: What's next in antidepressant drug development?

\title{
Therapeutic cloning gives silenced genes a second voice
}

As controversy continues on therapeutic cloning to create human embryos, applying the technique-also known as somatic cell nuclear transfer-in animals is generating important insights into disease development.

Some scientists are using the approach to study epigenetic alterations-chromosomal modifications that do not alter the DNA sequence-which can cause cancer. "A principal question in cancer research is what part of the cancer cell phenotype comes from genetic defects and what part is epigenetic," says Rudolf Jaenisch, professor of biology at the Whitehead Institute for Biomedical Research. "Nuclear transfer experiments can tell us that."

Therapeutic cloning involves injecting the nucleus of an adult cell into an egg that has had its own nucleus removed. Scientists have found that when the nucleus of a cancer cell is introduced into an egg, the cell's epigenetic features are stripped away-a process known as 'nuclear reprogramming.' "This is because epigenetic alterations, unlike genetic alterations, are reversible," says Jaenisch.

Once injected, the nucleus undergoes dramatic changes in structure and protein composition that somehow reset its genetic material and allow it to direct embryonic development, says Emory University geneticist Paul Wade.

One such modification, as indicated by recent experiments in Xenopus embryos, is the removal of methyl groups from specific regions of DNA. This may be a necessary step in the epigenetic reprogramming of the nucleus, the researchers suggest in the October Nature Cell Biology.

As cells differentiate, they accrue many other types of epigenetic alterations, such as the addition of phosphates or removal of acetyl groups from histones, or chromosomal proteins, and trigger changes in chromatin structure. Defects in these processes have been linked to cancer and other diseases.

Tumor suppressor genes are often methylated in cancer cells, notes Peter Jones, professor of biochemistry at the University of Southern California. Methylation keeps these genes turned off, promoting tumor growth, he says. "It has always been believed that once a gene becomes methylated, it is permanently silenced," Jones adds. "The $[$ Xenopus] findings show that this

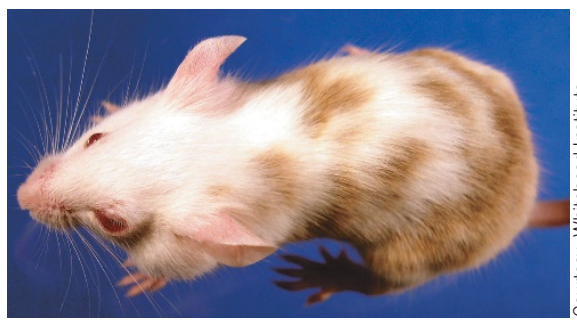

Reversing cancer: Scientists have cloned a norma mouse from a melanoma cell.

\section{silencing may not be permanent."}

Jaenisch and his colleagues have also shown that nuclei from a skin cancer cell can be reprogrammed to direct normal development of a mouse embryo-meaning that removal of the epigenetic alterations is enough to restore cells to normal (Genes Dev. 18, 1875-1885; 2004). An earlier study reported similar results with brain tumor cells (Cancer Res. 63, 2733-2736; 2003).

Based on such findings, pharmaceutical companies are racing to develop and test 'epigenetic therapies'-for example, drugs that prevent the addition of methyl groups might reactivate silenced tumor suppressor genes. Some such drugs are already in use to treat disorders such as myeloid dysplastic syndrome and sickle cell anemia and are being tested for some types of cancer. Compounds that can prevent histone deacetylation are also in early clinical trials for cancer and rheumatoid arthritis.

Because many normal cells continuously undergo epigenetic modifications, these drugs may have several toxic side effects. They are also unlikely to be effective in cancers whose origin is strictly genetic.

The primary benefit from these studies is in understanding disease mechanisms better, but the drugs hold some promise of therapeutic benefit. "If we can manipulate the human epigenome," says Wade, "we have the potential to influence a large number of human diseases."

Kris Novak, San Francisco

\section{US panel recommends 'black box' warnings for antidepressants}

Advisors to the US Food and Drug

Administration have asked the agency to add tough warnings to antidepressant medications, saying the drugs cause some children to become suicidal.

The advisory committee said it was concerned by data from 24 clinical trials showing that most antidepressant medications do not work in children. The data, most of which has not been published in peer-reviewed journals, also showed that these drugs as a class increase the risk of suicidal thoughts and behaviors in children.

"We have very strong evidence of harm and not good evidence of efficacy, and I know many practitioners are convinced these drugs work," said Thomas Newman, an epidemiologist and pediatrician at the University of California in San Francisco.

On 14 September, the committee voted 15 to 8 in favor of a so-called 'black box' warning that would inform patients and doctors about the risks.

Drug companies say the warning may scare away children who need the treatment. But the drugs' critics claimed victory after the hearing, saying the episode underscores the need for full disclosure of data from clinical trials.

The controversy over antidepressant use in children has accelerated the push for a clinical trial registry. On 8 September, the editors of 12 leading medical journals announced that trials published in their journals must be registered at the outset in a public database.

Their announcement came one day after the pharmaceutical industry trade organization PhRMA said it plans to set up a voluntary database where companies can post results from clinical trials. At a congressional hearing on 9 September, Eli Lilly executive John Hayes explained why the pharmaceutical industry is now eager to act: "There is clearly a societal crisis in terms of the credibility of drug company results," Hayes said.

Erika Check, Washington, DC 